\title{
Managing Immunotherapy-related Side Effects
}

\author{
Claire Friedman, $\mathrm{MD}^{1}$ and Michael Postow, MD² \\ 1. Fellow; 2. Medical Oncologist, Memorial Sloan Kettering Cancer Centre, New York, New York, us
}

DOI: http://doi.org/10.17925/OHR.2015.11.02.143

\begin{abstract}
This editorial on "Managing Immunotherapy-Related Side Effects" is based on an abstract presented at the American Society of Clinical Oncology Annual Congress, Chicago, IL, US, June 2015.

\section{Keywords}

Immunotherapy, immune-related adverse events, cytotoxic T-lymphocyte antigen 4 (CTLA-4) inhibitor, programmed cell death-1 (PD-1) inhibitor

Disclosure: Michael Postow, MD, has received honoraria from Bristol-Myers Squibb and Merck, research grants from Bristol-Myers Squibb, and engaged in Advisory Boards for Amgen and Bristol-Myers Squibb. Claire Friedman, MD, has nothing to declare in relation to this article. No funding was received in the publication of this article.

open Access: This article is published under the Creative Commons Attribution Noncommercial License, which permits any noncommercial use, distribution, adaptation, and reproduction provided the original author(s) and source are given appropriate credit.

Received: August 18, 2015 Published Online: November 10, 2015 Citation: Oncology \& Hematology Review, 2015;11(2):143-4

Correspondence Claire Friedman, MD, Memorial Sloan Kettering Cancer Center, 1275 York Ave, NY, NY 10065, US. E: friedmac@mskcc.org
\end{abstract}

The field of oncology immunotherapeutics has exploded recently with the development of immune-checkpoint inhibitors. These drugs enhance antitumor immunity by blocking negative regulators of T-cell function that exist both on immune cells and on tumor cells. There are two classes of immune-checkpoint inhibitors: currently the Food and Drug Administration (FDA) has approved cytotoxic T-lymphocyte antigen 4 (CTLA-4) antibodies ${ }^{1,2}$ and programmed cell death-1 (PD-1) antibodies for the treatment of metastatic melanoma. ${ }^{3,4}$ Nivolumab, a PD-1 inhibitor, has also been approved by the FDA for the treatment of metastatic squamous cell lung cancer. 5,6 These drugs are associated with unique inflammatory side effects known as immune-related adverse events (irAEs). Here, we present recommendations for the management of these events.

\section{Rash}

The most common irAE associated with checkpoint blockade is rash and/or pruritus. Nearly $50 \%$ of patients treated with the CTLA-4 inhibitor ipilimumab will experience this irAE. ${ }^{7}$ Rash is also one of the most common toxicities of anti-PD-1 therapy, but fortunately the rates of significant grade 3 and 4 rashes with these agents remain low. ${ }^{8,9}$ Rashes associated with checkpoint blockade typically appear faintly erythematous, reticular, and maculopapular, although there have been reports of atypical finding such as Sweet's syndrome and bullous pemphigoid. ${ }^{10,11}$ Topical corticosteroid creams can be used to treat rashes as well as oral antipruritics, such as benadryl and hydroxyzine for bothersome itching. Severe rash (grade 3 or above) should be treated with oral corticosteroids, usually at an equivalent dose of prednisone $1 \mathrm{mg} / \mathrm{kg}$ daily.

\section{Diarrhea/Colitis}

Diarrhea is also a common irAE of checkpoint blockade, although it is much more common with the CTLA-4-blocking antibodies compared with those targeting PD-19; the rate of grade 3/4 diarrhea associated with PD-1 blockade is only 1-2\%. ${ }^{8}$ While diarrhea and colitis lie along a similar clinical spectrum, in trials, they are often reported as separate toxicities based on different descriptions according to the Common Terminology Criteria for Adverse Events (CTCAE). According to the CTCAE, colitis is associated with abdominal pain and radiographic or endoscopic evidence of inflammation. Diarrhea more simply refers to an increase in stool frequency. The first step in management should always be to assess for other etiologies of diarrhea, such as infection with Clostridium difficile or other bacterial/viral pathogens. Once this has been ruled out, antidiarrheals, such as loperamide, can be utilized in mild cases. If symptoms persist or intensify, oral or intravenous corticosteroids should be implemented. For severe or steroid refractory symptoms, infliximab at a dose of $5 \mathrm{mg} / \mathrm{kg}$ has been utilized ${ }^{12-14}$ based on data from patients with inflammatory bowel disease. ${ }^{15}$ For patients who have a minimal increase in bowel movements over baseline that persists, budesonide can also be used. Unfortunately, there is no treatment that has been shown to prevent immune-checkpoint inhibitor diarrhea. Budesonide was tested as a prophylactic agent, but unfortunately it did not reduce the incidence of diarrhea. ${ }^{16}$

\section{Hepatotoxicity}

Hepatitis associated with checkpoint blockade is associated with elevations in aspartate transaminase (AST) and alanine transaminase (ALT) and, occasionally, bilirubin. In most cases, this is asymptomatic. Hepatic function should be monitored before each dose of checkpoint blockade, and, if elevated, viral and other drug-induced causes of hepatitis should be excluded. As with treating other irAEs, if no other immediate cause is obvious, prompt treatment with corticosteroids is recommended. In rare cases, elevations in AST and ALT are steroid-refractory and $500 \mathrm{mg}$ 
every 12 hours of mycophenolate mofetil may be helpful. Infliximab is contraindicated due to the risk of hepatotoxicity.

\section{Endocrinopathy}

Although the aforementioned irAEs usually have straightforward presentation, diagnosis of endocrinopathy associated with checkpoint blockade can be more challenging. Patients may present with nonspecific symptoms, including fatigue, nausea, and headache; therefore, practitioners must be vigilant. Hypophysitis (pituitary inflammation) and hypothyroidism are the most common endocrinopathies and are typically believed to occur in up to $10 \%$ of patients treated with CTLA-4 blockade. ${ }^{17,18}$ The frequency of endocrinopathy in patients treated with PD-1 or programmed death-ligand 1 agent is less well known, but appears to be less common. ${ }^{9}$

Typically, hypophysitis is diagnosed by clinical symptoms of fatigue and headache, radiographic findings (enhancement and enlargement of the pituitary ${ }^{19,20}$ ), and biochemical evidence of pituitary dysfunction (low adrenocorticotropic hormone and thyroid-stimulating hormone [TSH]). Some clinicians have described that a course of high-dose corticosteroids may reverse the inflammatory process, but most patients will require long-term supplementation with levothyroxine and/or replacement doses of hydrocortisone, such as $20 \mathrm{mg}$ each morning and $10 \mathrm{mg}$ each evening. Given that routine monitoring of thyroid function with a TSH laboratory evaluation is required during treatment with ipilimumab, patients may be diagnosed with thyroid function abnormalities before they are symptomatic. Hypothyroidism occurs far more commonly than hyperthyroidism and is managed with replacement doses of thyroid hormone.

\section{Pneumonitis}

Pneumonitis is a rare ( $<10 \%)$, but potentially life-threatening irAE seen in patients treated with CTLA-4- and PD-1-blocking agents. 3,4,2,21-23 Any patient presenting with pulmonary symptoms, such as an upper respiratory infection, new cough, or shortness of breath, should be assessed with imaging. In moderate to severe cases, a bronchoscopy should be performed to exclude infectious etiologies before starting immunosuppression. In severe cases, treatment should consist of high doses of corticosteroids such as $2 \mathrm{mg} / \mathrm{kg}$ of intravenous methylprednisolone and additional immunosuppression with agents such as infliximab maybe reasonable. No further doses of immune-checkpoint inhibition should be administered.

\section{Conclusion}

As immunotherapy emerges as a treatment modality for advanced solid tumors, oncology practitioners will be required to manage inflammatory side effects. Early recognition and aggressive treatment of grade 1-2 irAEs is recommended to prevent patient morbidity and mortality without compromising the efficacy of immune-checkpoint inhibition. Moving forward, it will be imperative to gather prospective data about use of both steroids and steroid-sparing agents to best develop treatment guidelines.
1. Hodi FS, O'Day SJ, MCDermott DF, et al., Improved survival with ipilimumab in patients with metastatic melanoma, $N$ Eng/ I Med, 2010;363:711-23.

2. Robert $\mathrm{C}$, Thomas $\mathrm{L}$, Bondarenko I, et al., Ipilimumab plus dacarbazine for previously untreated metastatic melanoma, N Engl J Med, 2011:364:2517-26.

3. Robert C, Long GV, Brady B, et al., Nivolumab in previously untreated melanoma without BRAF mutation, N Eng/ I Med, 2015;372:320-30

4. Robert C, Ribas A, Wolchok JD, et al., Anti-programmeddeath-receptor-1 treatment with pembrolizumab in ipilimumab-refractory advanced melanoma: a randomised dose-comparison cohort of a phase 1 trial, Lancet, 2014:384:1109-17.

5. Gettinger SN, Horn L, Gandhi L, et al., Overall survival and long-term safety of nivolumab (anti-programmed death 1 antibody, BMS-936558, ONO-4538) in patients with previously treated advanced non-small-cell lung cancer, $J$ Clin Oncol, 2015;33:2004-12

6. Brahmer J, Reckamp KL, Baas P, et al., Nivolumab versus docetaxel in advanced squamous-cell non-small-cell lung cancer, N Engl I Med, 373:123-35.

7. Weber JS, Kähler KC, Hauschild A, Management of immunerelated adverse events and kinetics of response with ipilimumab, J Clin Oncol, 2012;30:2691-97.

8. Topalian SL, Sznol M, MCDermott DF, et al., Survival, durable tumor remission, and long-term safety in patients with advanced melanoma receiving nivolumab, J Clin Oncol, 2014;32:1020-30

9. Robert C, Schachter J, Long GV, et al., Pembrolizumab versus Ipilimumab in advanced melanoma, N Eng/ I Med, 2015:372:2521-32.

10. Pintova S, Sidhu H, Friedlander PA, Holcombe RF, Sweet's syndrome in a patient with metastatic melanoma after ipilimumab therapy, Melanoma Res, 2013;23:498-501.

11. Carlos $G$, Anforth R, Chou S, et al., A case of bullous pemphigoid in a patient with metastatic melanoma treated with pembrolizumab, Melanoma Res, 2015;25:265-8.

12. Pages $\mathrm{C}$, Gornet $\mathrm{JM}$, Monsel $\mathrm{G}$, et al., Ipilimumab-induced acute severe colitis treated by infliximab, Melanoma Res, 2013:23:227-30.

13. Merrill SP, Reynolds P, Kalra A, et al., Early administration of infliximab for severe ipilimumab-related diarrhea in a critically il patient, Ann Pharmacother, 2014;48:806-10.

14. Minor DR, Chin K, Kashani-Sabet M, Infliximab in the treatment of anti-CTLA4 antibody (ipilimumab) induced immune-related colitis, Cancer Biother Radiopharm, 2009;24:321-5.

15. Stidham RW, Lee TC, Higgins PD, et al., Systematic review with network meta-analysis: the efficacy of anti-TNF agents for network meta-analysis: the efficacy of anti-TNF agents for the treatment of

16. Weber J, Thompson JA, Hamid O, et al., A randomized, doubleblind, placebo-controlled, phase II study comparing the tolerability and efficacy of ipilimumab administered with or without prophylactic budesonide in patients with unresectable stage III or IV melanoma, Clin Cancer Res, 2009;15: 5591-8.

17. Corsello SM, Barnabei A, Marchetti P, et al., Endocrine side effects induced by immune checkpoint inhibitors, $J$ Clin Endocrinol Metab, 2013:98:1361-75.

18. Ryder M, Callahan M, Postow MA, et al., Endocrine-related adverse events following ipilimumab in patients with advanced melanoma: a comprehensive retrospective review from a single institution, Endocr Relat Cancer, 2014;21:371-81.

19. Blansfield JA, Beck KE, Tran K, et al., Cytotoxic T-lymphocyteassociated antigen-4 blockage can induce autoimmune hypophysitis in patients with metastatic melanoma and renal cancer, J Immunother, 2005:28:593-8.

20. Dillard T, Yedinak CG, Alumkal J, Fleseriu M, Anti-CTLA-4 antibody therapy associated autoimmune hypophysitis: serious immune related adverse events across a spectrum of cancer subtypes, Pituitary, 2010;13:29-38.

21. Hamid O, Schmidt H, Nissan A, et al., A prospective phase II trial exploring the association between tumor microenvironment biomarkers and clinical activity of ipilimumab in advanced melanoma, J Trans/ Med, 2011:9:204.

22. Topalian SL, Hodi FS, Brahmer JR, et al., Safety, activity, and immune correlates of anti-PD-1 antibody in cancer, N Eng/ 」 Med, 2012;366:2443-54.

23. Larkin J, Chiarion-Sileni V, Gonzalez R, et al., Combined nivolumab and ipilimumab or monotherapy in untreated melanoma, N Eng/ I Med, 2015;373:23-34. 